\title{
THE HYDRATION OF AN ION-LEACHABLE GLASS USED IN GLASS-IONOMER CEMENTS
}

\author{
JACEK KŁOS*, BEATA CZARNECKA**, ${ }^{\#}$ JOHN W. NICHOLSON*** \\ *Department of Physical Chemistry, Faculty of Chemistry, Adam Mickiewicz University of Poznań, \\ ul. Umultowska 89b, 61-614 Poznań, Poland \\ **Department of Biomaterials and Experimental Dentistry, Dental Institute, University of Medical Sciences, \\ ul Bukowska 70, 60-812 Poznań, Poland \\ ***Bluefield Centre for Biomaterials, 67-68 Hatton Garden, London, EC1N 8JY, United Kingdom \\ "E-mail: john.nicholson@bluefieldcentre.co.uk
}

Submitted April 1, 2016; accepted June 10, 2016

Keywords: Glass-ionomers, Hydration, Compressive strength, Raman spectroscopy

\begin{abstract}
A study is reported in which the interaction between a typical ionomer glass and water was evaluation in order to evaluate the importance of hydration in the setting of glass-ionomer cements. Glass G338 was mixed with water and the slurries were allowed to harden in metal moulds to create cylindrical specimens $6 \mathrm{~mm}$ high $\times 4 \mathrm{~mm}$ diameter. Samples of these specimens were found to disintegrate when placed in water. Following hardening at $37^{\circ} \mathrm{C}$ for 1 hour in the moulds, one series of specimens was stored at $95 \%$ RH for $23 \mathrm{~h}, 1$ week and 4 weeks, and the other stored for the same lengths of time, but sealed in the moulds. Raman spectra were recorded for glass G338 and glass-water blends stored for 24 hours and 4 weeks. The cylindrical specimens were found to have a degree of structural integrity, but proved to be extremely weak in compression (all specimens of whatever age up to 4 weeks having strengths of less that $1 \mathrm{MPa}$ ). Specimens lost mass on storage at $95 \%$ RH. Raman spectra showed no additional bands due to glass-water interactions compared with the dry glass itself, and changes in intensity were difficult to interpret, due to Fermi resonance in the regions of interest. It is concluded that binding in these specimens is due to hydrogen bonding between layers of water adsorbed onto glass powder surfaces.
\end{abstract}

\section{INTRODUCTION}

Glass-ionomer cements are widely used materials in dentistry for the repair of teeth damaged by caries, with applications including liners and bases, and full restorations [1]. They are also used preventively as pitand-fissure sealants [2].

The setting of glass-ionomer cements has been studied extensively, and many aspects of this are well established. The initial step is attack on the glass by protons from the acid [3], leading to ion release that is closely followed by formation of metal carboxylate groups [4, 5]. These groups act as crosslinks between the polymer chains, and the effect of this, coupled with the coil expansion of the polyanion, lead to hardening of the cement [6].

Following this setting process, there is a series of post-hardening reactions that are generally described as maturation. Various processes are known to take place, including an increase in the proportion of bound water within the cement [7] and an improvement in the translucency [8]. The detailed mechanisms of these processes are not known, though it is known that the proportion of 6-coordinate aluminium increases relative to the amount of 4-coordinate [9].
The means by which water becomes bound into maturing glass-ionomer cements has received little attention so far. It has been suggested that it may progressively coordinate metal ions, such as $\mathrm{Ca}^{2+}$ and $\mathrm{Al}^{3+}[10]$ and also occupy regions close to the polyanion molecule [11]. FTIR studies have shown that silanol groups are present in set cements [12], and these presumably arise from hydration of silicate structures in the surface of the glass. Such processes are known with silica and other types of silicate glass $[13,14]$ and seem plausible in the glasses used in glass-ionomer cements.

These glasses are known to release small amounts of ions when exposed to dilute acetic acid solutions $[15,16]$. This release was found to lead to the formation of small amounts of friable inorganic solid in the surface of water in contact with ionomer glass powder [15]. The reaction was also shown to be capable of creating cements that were insoluble in water after 24 hour [16], and which eventually became reasonably strong in compression [17].

These results show that glasses used in glass-ionomer cements are capable of forming cements without any polymer being present. However, the role of water in this setting this has not been studied to any extent. It is known to be the solvent for the setting reaction, and to 
become fully incorporated into the set cement without phase separation [18]. However, no other aspects have been considered.

In the current paper, we address the question of whether ionomer glass can undergo hydrolysis and whether the product of such a reaction has any mechanical integrity. Using the glass G338, a glass of the type that is widely used in commercial glass-ionomers designed for use in clinical dentistry $[15,16]$, we have mixed glass with water and used the resulting slurry to fabricate test pieces. These specimens have been tested for strength in compression, and changes in strength with time have been determined. Results give insight into the significance of simple hydration processes in these materials.

\section{EXPERIMENTAL}

The glass employed in this study was G338, (obtained from First Scientific Dental, Elmshorn, Germany), the pre-firing composition of which is shown in Table 1. This was mixed with deionised water at two different powder:water ratios by mass, namely 3.75 : and 3.5:1. Powder and water were prepared into slurries by spatulating them together at the appropriate ratio on a ceramic tile using a metal mixing spatula.

Small amounts of set slurry were allowed to remain for up to 24 hours, then tested for solubility by placing in water and observing the effect

Compressive strength of hydrated G338 specimens were determined on six specimens for each cement, each specimen being a cylinder of size $6 \mathrm{~mm}$ height by $4 \mathrm{~mm}$ diameter. They were prepared in split metal moulds, and allowed to harden in the moulds for 1 hour at $37^{\circ} \mathrm{C}$. One set of specimens for each powder:water ratio was then tested in compression immediately.

Further determinations of compressive strength were carried out on sets of specimens that were either stored sealed in their moulds for time periods of 23 hours, 1 week and 4 weeks or removed from the moulds and stored at $95 \%$ RH for 23 hours, 1 week and 4 weeks. For the latter, mass changes were determined by weighing the specimens on removal from the mould

Table 1. Pre-firing composition of glass G338.

\begin{tabular}{lr}
\hline Component & $\%$ \\
\hline $\mathrm{SiO}_{2}$ & 24.9 \\
$\mathrm{Al}_{2} \mathrm{O}_{3}$ & 14.2 \\
$\mathrm{Na}_{3} \mathrm{AlF}_{6}$ & 19.2 \\
$\mathrm{CaF}_{2}$ & 12.8 \\
$\mathrm{AlF}_{3}$ & 4.6 \\
$\mathrm{AlPO}_{4}$ & 24.2 \\
Other oxides & 0.1 \\
\hline
\end{tabular}

The material was ground to a fine powder, mean particle size approximately $4 \mu \mathrm{m}(87.7 \%$ at $10 \mu \mathrm{m}$ or less $)$. and after the appropriate storage period. For storage outside the moulds, specimens were stored in a sealed chamber maintained at $95 \%$ RH using saturated sodium sulfate solution [19].

In all cases, compression testing was carried out using a universal testing machine (Hounsfield H5KS, Redhill, Surrey, UK) and a crosshead speed of $1.0 \mathrm{~mm} \cdot \mathrm{min}^{-1}$. Loads at failure were converted to compressive strength values, and means and standard deviations were determined for each composition and for each maturation period. Differences in compressive strength and mass change were assessed for significance by 1-way ANOVA followed by the Student t-test.

Finally, Raman spectra were determined in specimens after 23 hours and 4 weeks storage at $95 \% \mathrm{RH}$. In addition, the Raman spectrum of glass G338 was recorded. Spectra were recorded using a Bruker RFS 100/S spectrometer (Bruker, Leipzig, Germany).

\section{RESULTS}

The specimens all had an obvious degree of integrity on removal from the moulds. They retained their shape, and could be easily transferred to the universal testing machine for further testing.

Compressive strength data are shown in Table 2. Values differed significantly $(p>0.01)$ for the two different powder:water ratios stored for 1 hour. In both cases, strengths at 24 hours after storage at $95 \% \mathrm{RH}$ did not differ significantly. This result came about because the 3.5: specimens at 1 hour and 24 hours did not differ in compressive strength, whereas the $3.75: 1$ specimens increased in compressive strength by a significant amount over this time.

After 1 week at $95 \% \mathrm{RH}$, compressive strengths fell by amounts that were significant $(p<0.01)$. They apparently rose again slightly by 4 weeks, but these changes were not significant.

For the specimens stored for 24 hours at $95 \% \mathrm{RH}$, there was a decrease in mass in all cases (Table 3), with extent of mass loss varying with storage time. This shows that these cements were able to lose water, even in high humidity conditions, though this water loss did not differ significantly between the two powder:water ratios at any time interval. By contrast, the change between 23 hours and 1 week was significant $(p<0.01)$ in both cases.

Specimens stored sealed in their moulds were weaker in compression at 24 hours than they were at 1 hour, showing that some sort of changes occurred in the specimens that did not involve water loss. From 24 hours onwards, compressive strengths did not vary significantly either with time or between powder : water ratios.

Raman spectroscopy data are given in Table 4. Similar bands occurred in G338 powder, and G338water blends aged for 24 hours and 4 weeks, with only minor differences in intensity. 
The hydration of an ion-leachable glass used in glass-ionomer cements

Table 2. Compressive strength of G338-water blends (Standard deviations in parentheses).

\begin{tabular}{|c|c|c|c|}
\hline $\begin{array}{c}\text { Specimen } \\
\text { (glass : water ratio, mass : mass) }\end{array}$ & Storage time & Storage conditions & $\begin{array}{c}\text { Compressive strength } \\
(\mathrm{MPa})\end{array}$ \\
\hline $3.5: 1$ & 1 hour & In mould & $0.820(0.049)$ \\
\hline $3.75: 1$ & 1 hour & In mould & $0.646(0.109)$ \\
\hline $3.5: 1$ & 23 hours & $95 \% \mathrm{RH}$ & $0.930(0.144)$ \\
\hline $3.75: 1$ & 23 hours & $95 \% \mathrm{RH}$ & $0.893(0.097)$ \\
\hline $3.5: 1$ & 1 week & $95 \% \mathrm{RH}$ & $0.283(0.056)$ \\
\hline $3.75: 1$ & 1 week & $95 \% \mathrm{RH}$ & $0.302(0.075)$ \\
\hline $3.5: 1$ & 4 weeks & $95 \% \mathrm{RH}$ & $0.430(0.073)$ \\
\hline $3.75: 1$ & 4 weeks & $95 \% \mathrm{RH}$ & $0.464(0.108)$ \\
\hline $3.5: 1$ & 23 hours & In mould & $0.488(0.166)$ \\
\hline $3.75: 1$ & 23 hours & In mould & $0.353(0.128)$ \\
\hline $3.5: 1$ & 1 week & In mould & $0.310(0.158)$ \\
\hline $3.75: 1$ & 1 week & In mould & $0.382(0.120)$ \\
\hline $3.5: 1$ & 4 weeks & In mould & $0.348(0.143)$ \\
\hline $3.75: 1$ & 4 weeks & In mould & $0.387(0.109)$ \\
\hline
\end{tabular}

Table 3. Mass loss on storage at $95 \%$ RH (Standard deviations in parentheses).

\begin{tabular}{ccc}
\hline $\begin{array}{c}\text { Specimen } \\
\text { (glass : water ratio, mass : mass) }\end{array}$ & $\begin{array}{c}\text { Storage } \\
\text { time }\end{array}$ & $\begin{array}{c}\text { Mass loss } \\
(\%)\end{array}$ \\
\hline $3.5: 1$ & $23 \mathrm{~h}$ & $5.21(1.00)$ \\
$3.75: 1$ & $23 \mathrm{~h}$ & $4.33(1.56)$ \\
$3.5: 1$ & 1 week & $15.78(0.51)$ \\
$3.75: 1$ & 1 week & $14.42(0.74)$ \\
$3.5: 1$ & 4 weeks & $16.53(0.29)$ \\
$3.75: 1$ & 4 weeks & $15.65(0.76)$ \\
\hline
\end{tabular}

\section{DISCUSSION}

For the first time, we report that the ionomer glass G338 is capable of forming a substance with a degree of structural integrity simply on reaction with water. Previous studies have shown that this glass will form what have been termed "pseudo-cements" with monomeric acids such as acetic and lactic acid [17, 20], but what we report here is different from these substances in that it remains soluble in water. The product of hydration is therefore not a cement of any kind. However, it did not slump on standing outside the moulds, and had a measurable if low compressive strength, showing that there is some sort of connection between the glass particles in these blends.

Earlier studies have used acetic and lactic acids at concentrations of $40-50 \%$ (for acetic acid) [17] and $25-75 \%$ (for lactic acid) [20] to form cements, and these seem to develop their insolubility from the formation of some sort of inorganic network, probably based on phosphates [21]. Compressive strength of these pseudo-cements generally increased with increasing concentration of acid, with values falling between 4.8 and $35.1 \mathrm{MPa}$ for specimens aged for 24 hours. These are substantially stronger than the materials formed by reaction of G338 with water, all values of which fell below $1 \mathrm{MPa}$.
Storage of the G338-water materials in $95 \% \mathrm{RH}$ led to loss of mass, which must be due to loss of water to the atmosphere, despite the high humidity conditions. This loss varied with time, and shows that these glasswater materials have a substantial amount of evaporable water within them. However, in no case was all of the water lost, showing that the G338 glass has a reasonably strong affinity for water. The overall compressive strength was affected by this water loss, falling at longer storage times as more water was lost from the materials.

Details of the Raman spectra for three key materials, G338 itself and G338-water (powder:water ratio: 3.5:1) stored for 24 hours and 4 weeks are shown in Table 4. Intensities differed only slightly between these three materials, and similar bands were present in all cases. Some bands clearly originate in the glass. For example, the band at $1072-74 \mathrm{~cm}^{-1}$ can be attributed to Q3 $\mathrm{Si}-\mathrm{O}$ species, since such a band has previously been reported as occurring at about $1100 \mathrm{~cm}^{-1}$ [22] and at $1083 \mathrm{~cm}^{-1}$ [23] in similar silicate substances. In the same way, the bands at $2692-2715 \mathrm{~cm}^{-1}$ and $2534-2538 \mathrm{~cm}^{-1}$ are in the regions where bands have previously been observed in the spectra of hydrous sodium silicate glasses [24].

The bands in the region $2860-2867 \mathrm{~cm}^{-1}$ can tentatively be assigned to strong hydrogen-bonds between silanol groups on the glass surface and molecular water [25]. This suggests that even "dry" G338 powder has a surface film of water, which is possible, but not completely confirmed by the Raman spectroscopy results, since there is no well-defined peak in the region of $1630 \mathrm{~cm}^{-1}$, the known position of the band from liquid water [22]. Unfortunately, bands in these regions appear to be caused by Fermi resonance between fundamental bands [25], which complicates peak intensities, and means that assigning their origin is difficult [25]. It also means that there is no simple correlation between intensity of the peaks and relative amount of substance which give rise to absorptions in this region. This means that we cannot simply compare spectra from the G338water blends with that from G338 alone. 
Kłos J., Czarnecka B., Nicholson J. W.

Table 4. Main Raman bands $\left(\mathrm{cm}^{-1}\right)$ in the spectra of glass G338 and G338-water samples.

\begin{tabular}{|c|c|c|c|}
\hline \multirow[t]{2}{*}{ G338 only } & \multicolumn{2}{|c|}{ G338-water (3.5: 1 , mass : mass) } & \multirow[t]{2}{*}{ Description } \\
\hline & stored for $24 \mathrm{~h}$ & stored for 4 weeks & \\
\hline 3252 & 3252 & 3253 & Broad, medium \\
\hline 2866 & 2867 & 2860 & Broad, weak \\
\hline 2692 & 2694 & 2715 & Weak \\
\hline 2654 & 2658 & 2655 & Weak \\
\hline 2615 & 2618 & 2618 & Weak \\
\hline 2536 & 2534 & 2538 & Weak \\
\hline 2198 & 2197 & 2196 & Medium \\
\hline 2125 & 2125 & 2125 & Medium \\
\hline 1961 & 1961 & 1957 & Medium \\
\hline 1590 & 1590 & 1600 & Medium \\
\hline 1284 & 1289 & 1294 & Medium \\
\hline 1073 & 1072 & 1074 & Medium \\
\hline
\end{tabular}

As a consequence, the Raman spectra gave less insight into the bonding of these materials than hoped, and the results do not allow an unambiguous explanation to be offered for the structural integrity of these materials. However, it seems highly likely that silanol groups on the glass surface interacting with molecular water is responsible for the weak structure of these materials, via hydrogen bonding. This possibility requires further work to confirm in the current materials.

\section{CONCLUSIONS}

The ionomer glass G338 mixed with water has been found to set to form materials with a degree of integrity. Structures formed in this process have modest mechanical strength in compression, though they are much weaker than actual glass-ionomer cements or pseudo-cements formed by this glass with either acetic or lactic acid. These materials probably arise from silanol groups on the surface of the glass, and their interaction with molecular water. Such hydration does not seem to be significant in contributing to the strength of either in pseudo-cements or true glass-ionomers, because the resulting material is water-soluble and mechanically weak. However, it may be significant in providing a site within the material at which water can collect, where it may act as the source of water for further maturation.

\section{REFERENCES}

1. Mount G.J. (2002). Color atlas of glass ionomer cement. $2^{\text {nd }}$ ed. Martin Dunitz.

2. Lindermeyer R.G. (2007): The use of glass ionomer sealants on newly erupting permanent molars. Journal of the Canadian Dental Association, 73, 131-134.

3. Prentice L. H., Tyas M. J., Burrow, M. F. (2007): Ion leaching of a glass-ionomer glass: an empirical model and effects on setting characteristics and strength. Journal of Materials Science: Materials in Medicine, 18(1), 127-131. doi:10.1007/s10856-006-0670-0
4. Crisp S., Pringuer M. A., Wardleworth D., Wilson A. D. (1974): Reactions in glass ionomer cements: II. An infrared spectroscopic study. Journal of dental research, 53(6), 1414-1419. doi:10.1177/00220345740530062001

5. Nicholson J.W., Brookman P.J., Lacy O.M., Wilson A.D. (1988): The influence of (+)-tartaric acid on the setting of glass-ionomer dental cements. Journal of dental research, 67(12), 1451-1454. doi:10.1177/00220345880670120201

6. Crisp S., Wilson A. D. (1974): Reactions in glass ionomer cements: III. The precipitation reaction. Journal of dental research, 53(6), 1420-1424. doi:10.1177/00220345740530 062101

7. Wilson A. D., Crisp S., Paddon J. M. (1981): The hydration of a glass-ionomer (ASPA) cement. British Polymer Journal, 13(2), 66-70. doi:10.1002/pi.4980130206

8. Asmussen E. (1983): Opacity of glass-ionomer cements. Acta Odontologica Scandinavica, 41(3), 155-157. doi:10.3109/00016358309162317

9. Zainuddin N., Karpukhina N., Hill R. G., Law R. V. (2009): A long-term study on the setting reaction of glass ionomer cements by ${ }^{27} \mathrm{~A} 1 \mathrm{MAS}-\mathrm{NMR}$ spectroscopy. dental materials, 25(3), 290-295. doi:10.1016/j.dental.2008.07.008

10.Wilson A.D., Nicholson J.W. (1993). Acid-base cements. Camridge University Press.

11. Ikegami A. (1964): Hydration and ion binding of polyelectrolytes. Journal of Polymer Science Part A: General Papers, 2(2), 907-921. doi: 10.1002/pol.1964.100020226

12. Matsuya S., Maeda T., Ohta M. (1996): IR and NMR analyses of hardening and maturation of glass-ionomer cement. Journal of dental research, 75(12), 1920-1927. do i: $10.1177 / 00220345960750120201$

13.Zhuravlev L. T. (2000): The surface chemistry of amorphous silica. Zhuravlev model. Colloids and Surfaces A: Physicochemical and Engineering Aspects, 173(1), 1-38. doi:10.1016/S0927-7757(00)00556-2

14. Tadjiev D. R., Hand R. J. (2010): Surface hydration and nanoindentation of silicate glasses. Journal of Non-Crystalline Solids, 356(2), 102-108. doi:10.1016/j.jnoncrysol. 2009.10.005

15. Wilson, A. D. (1996): Secondary reactions in glassionomer cements. Journal of materials science letters, 15(3), 275-276. doi:10.1007/BF00274473

16. Wasson E. A., Nicholson J. W. (1991): Studies on the setting chemistry of glass-ionomer cements. Clinical materials, 7(4), 289-293. doi:10.1016/0267-6605(91)90072-N 
17. Wasson E. A., Nicholso, J. W. (1993): New aspects of the setting of glass-ionomer cements. Journal of dental research, 72(2), 481-483. doi:10.1177/00220345930720020 201

18. Nicholson J. W. (1998): Chemistry of glass-ionomer cements: a review. Biomaterials, 19(6), 485-494. doi:10.1016/ S0142-9612(97)00128-2

19. Lange N.A. (1961). Handbook of chemistry. $10^{\text {th }} \mathrm{ed}$, McGraw-Hill, New York.

20. Nicholson J. W., Tawfik H., Czarnecka B. (2002): A study of cements formed by aqueous lactic acid and aluminosilicate glass. Journal of Materials Science: Materials in Medicine, 13(4), 417-419. doi:10.1023/A:1014305105445

21. Shahid S., Billington R. W., Pearson G. J. (2008): The role of glass composition in the behaviour of glass acetic acid and glass lactic acid cements. Journal of Materials Science:
Materials in Medicine, 19(2), 541-545. doi:10.1007/ s10856-007-0160-Z

22. McMillan P. F., Remmele R. L. (1986): Hydroxyl sites in $\mathrm{SiO}_{2}$ glass: A note on infrared and Raman spectra. American Mineralogist, 71(5-6), 772-778.

23. Kalampounias A. G. (2011): IR and Raman spectroscopic studies of sol-gel derived alkaline-earth silicate glasses. Bulletin of Materials Science, 34(2), 299-303. doi:10.1007/ s12034-011-0064-x

24. Shelby J. E., McVay G. L. (1976): Influence of water on the viscosity and thermal expansion of sodium trisilicate glasses. Journal of Non-Crystalline Solids, 20(3), 439-449. doi:10.1016/0022-3093(76)90124-1

25. Zarubin D. P. (1999): Infrared spectra of hydrogen bonded hydroxyl groups in silicate glasses. A re-interpretation. Physics and chemistry of glasses, 40(4), 184-192. 